\title{
Diagnóstico prenatal de inversión pericentrica del cromosoma cinco de novo en una paciente con gestación a término sin complicaciones posteriores
}

\author{
Prenatal diagnosis of pericentric de novo inversion in the fifth \\ chromosome in a patient with term birth without posterior complications \\ Carolina Torres ${ }^{1}$, Carla Usta ${ }^{1}$, Laura Mancilla ${ }^{1}$, Isabel Fernández ${ }^{2}$, Luis Gustavo Celis ${ }^{1}$
}

\section{Resumen}

Los avances en las técnicas de citogenética han permitido detectar con mayor precisión alteraciones cromosómicas tanto estructurales como de número. La amniocentesis genética es una prueba invasiva que se realiza entre la semana 16 y 20 de gestación que nos permite detectar distintas alteraciones cromosómicas. Presentamos un caso de una paciente que se le realizó a las 18 semanas de gestación la amniocentesis por edad materna avanzada (39 ańos), evidenciándose en el cariotipo una inversión pericéntrica del cromosoma 5. Se procedió a realizar cariotipos a los padres, ambos normales. De acuerdo con este resultado a la paciente se le realizó ecosonogramas para detectar si el feto presentaba malformaciones y se realizó asesoramiento genético. A continuación, se hizo evaluación del recién nacido y seguimiento durante 4 años para evaluar fenotipo y desarrollo neurológico. Como se comentará, el cromosoma 5 codifica para muchos genes y es responsable de muchas patologías, de las cuales este paciente no presentó ninguna.

Palabras claves: cromosoma 5, inversión pericéntrica, anomalías, cariotipo, genes, amniocentesis.

\begin{abstract}
Advances in cytogenetic techniques have made it possible to, more accurately, detect both structural and number chromosomal alterations. Genetic amniocentesis is an invasive test that is performed between week 16 and 20 of gestation that allows us to detect chromosomal alterations. We present a case of a patient who underwent amniocentesis by advanced maternal age (39 years) at 18 weeks of gestation, showing a pericentric inversion of chromosome 5 in the karyotype and proceeded to perform karyotypes of the parents, both normal. According to this result, the patient was screened for fetal malformations and genetic counseling. Newborn evaluation and 4-year follow-up to assess phenotype and neurological development. As discussed, chromosome 5 codes for many genes and is responsible for many pathologies that this patient did not present.
\end{abstract}

Keywords: chromosome 5, inversion pericentric, anomalies, karyotype, genes, Amniocentesis. 


\section{Introducción}

Dados los avances científicos en medicina, el ser humano puede acceder a diferentes métodos diagnósticos; la amniocentesis es uno de esos métodos ampliamente usado mediante el cual se puede realizar un cariotipo en el líquido amniótico para diagnosticar diferentes alteraciones a nivel genético (1-2).

El cromosoma 5 es uno de lo mas grandes pares en los 23 pares cromosomicos en los seres humanos, representando un $6.08 \%$ del $\mathrm{ADN}$ total (3), el cual posee aproximadamente 923 genes, algunos de los cuales están implicados en la formación de moléculas de transmisión de señales inmunes (4-5).

Las inversiones cromosómicas son un tipo común de estructura equilibrada, se clasifican en paracéntricas y pericéntricas. Hay datos que muestran que la inversión paracéntrica se puede hallar de 10 a 50 en cada 100.000 personas, en cambio la pericéntrica se ha encontrado en 12 a 70 por cada 100.000 personas (6). Generalmente, las inversiones no muestran ninguna alteración fenotípica; sin embargo, esa reorganización cromosómica puede resultar en gametos no balanceados $(5,7)$.

En este artículo reportamos un caso de una inversión del cromosoma 5 de diagnostico prenatal, evaluación de recién nacido de 39 semanas, y seguimiento durante 4 años dadas las múltiples enfermedades posnatales que pueden generarse en esta alteración. El caso de este paciente complementa otros reportes ilustrando que a pesar de una inversión del cromosoma 5 existe la posibilidad de un desarrollo normal en el niño.

\section{Métodos}

Se realizó amniocentesis genética a las 18 semanas de gestación por indicación de su médico tratante, riesgo sérico y edad materna avanzada (E.M.A). Se recibieron $20 \mathrm{cc}$ de líquido amniótico, claro, punción única, sin complicaciones. Se procedió a rea- lizar el cultivo celular en dos frascos de cultivo de $25 \mathrm{~cm} 3$ utilizando el medio de cultivo Chang Amnio (Irvine Scientific), luego fueron colocados en incubadora de $\mathrm{CO} 2$, realizando cambios de medio de cultivo y posteriormente se realizó la cosecha para la obtención de los cromosomas. Posteriormente se realizó el análisis de 20 metafases mediante la técnica de Bandas G.

Se realizó cariotipo a los padres para determinar la posibilidad de que alguno de ellos fuera portador sano de la inversión, siendo ambos normales.

Se realizó asesoramiento genético y se le indica evaluación del recién nacido.

\section{Resultados}

Se trata de un recién nacido a término con madre de 39 ańos primigestante ( ver Figura 1), en quien se consideró pertinente realización de cariotipo en líquido amniótico a las 18 semanas de embarazo por parte del médico tratante con indicación riesgo sérico en el primer trimestre y edad materna avanzada (EMA). Los padres eran saludables sin consanguinidad y sin antecedentes de enfermedades genéticas hereditarias hasta tres generaciones, con resultado de cariotipo dentro de límites normales (46, XX - 46, XY). Embarazo a término controlado, sin complicaciones. A la gestante se le realizó controles prenatales completos dentro de límites normales.

Figura 1. Árbol genealógico del paciente.

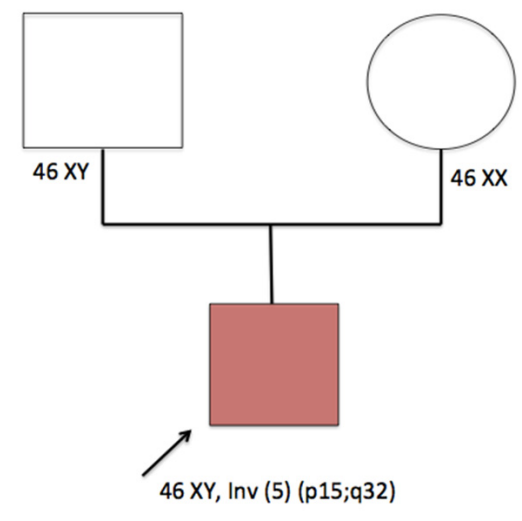

Fuente. Elaboración propia. 
El resultado de cariotipo realizado en el líquido amniótico producto de la amniocentesis evidenció paciente masculino anormal con inversión peri- céntrica del cromosoma 5 de novo. 46, $X Y$, inv (5) ( $15 ; q 32)$, posteriormente a este resultado se realizó asesoramiento genético (ver Figura 2).

Figura 2. Cariotipo 46, XY, (inv) 5 (p15,q32) [metafase 20].

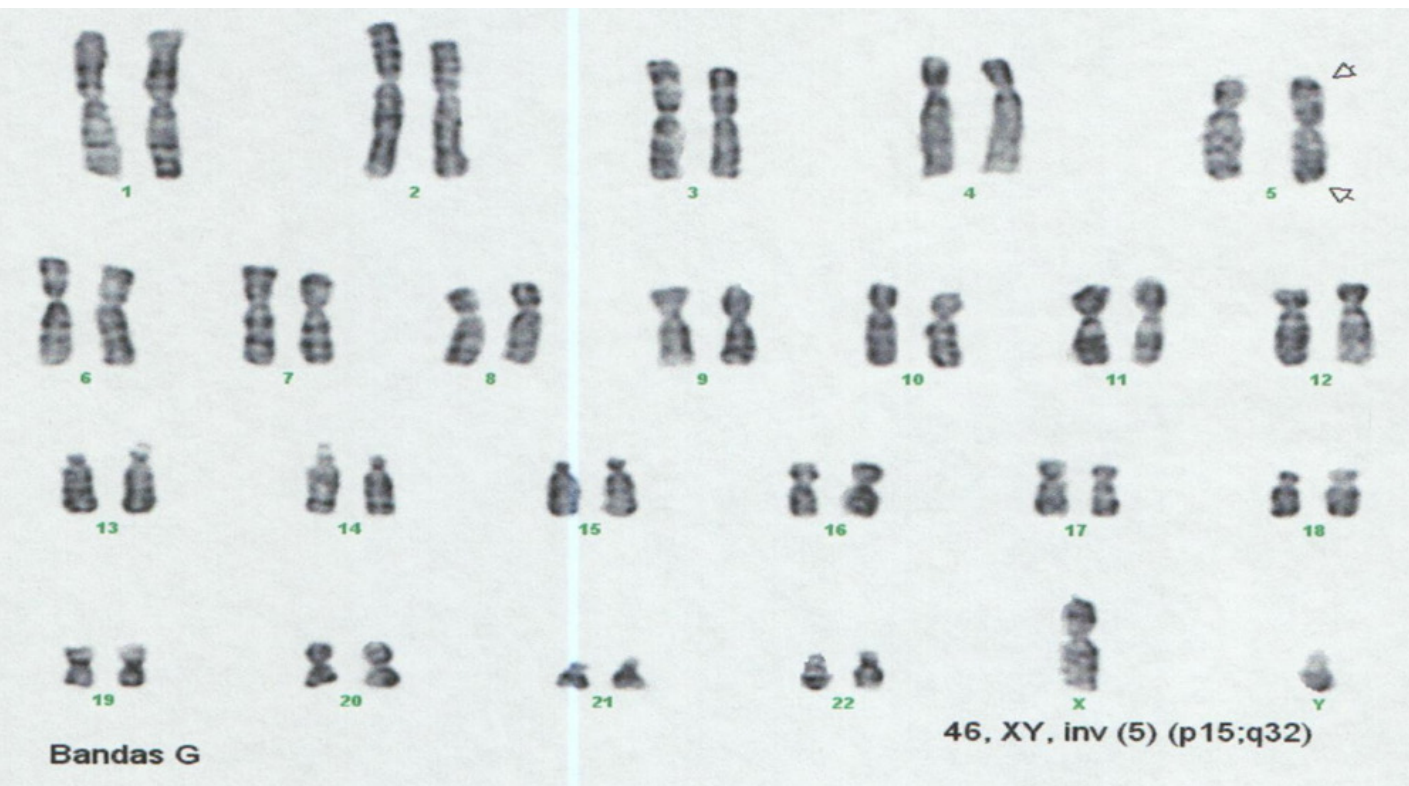

Fuente. Elaboración propia.

$\mathrm{Al}$ nacimiento se recibió recién nacido vivo de sexo masculino vía abdominal (cesárea) sin complicaciones, adecuada adaptación neonatal, sin requerimiento de oxígeno suplementario ni soporte vasopresor, peso adecuado para la edad gestacional, fenotipo normal; en quien se decidió alojamiento conjunto sin complicaciones y al segundo día alta hospitalaria.

Se continuó seguimiento, actualmente (2017) paciente con 4 años de edad con curvas de crecimiento y desarrollo neurológico dentro de límites normales (no déficit cognitivo o motor, a pesar de alteración cromosómica evidenciada en el cariotipo).

\section{Discusión}

En este artículo se ha descrito un paciente que presenta una inversión pericéntrica del cromosoma 5 de novo en un recién nacido a término sin altera- ciones fenotípicas ni del desarrollo, que podría estar asociado a múltiples enfermedades.

Los seres humanos normalmente tienen 46 cromosomas divididos en 23 pares, donde existe dos copias del cromosoma 5, el cual abarca cerca de 181 millones de bloques de DNA representando así casi el $6 \%$ del total de DNA en las células. Se considera que el cromosoma 5 contiene cerca de 900 genes que dan instrucciones para la generación de varias proteínas las cuales tendrán múltiples roles en el cuerpo humano. Por lo tanto, diferentes cambios en la estructura del cromosoma 5 llevarían a múltiples efectos incluyendo retraso en el crecimiento y desarrollo, alteraciones de la estructura facial, defectos al nacimiento y otros problemas de salud. (8).

Algunas de las alteraciones que se pueden presentar en el cromosoma 5 incluyen un segmento extra del brazo corto (p) o del brazo largo (q) del cromosoma 
en cada célula; La pérdida de un segmento del brazo largo, alteración en anillo del cromosoma, translocaciones e inversiones como se expuso en este caso, cada una de las cuales puede generar múltiples alteraciones y enfermedades (ver Figura 3) (8-9).

Las inversiones cromosómicas son un tipo común de estructura equilibrada humana las cuales se clasifican en paracéntrica y pericéntrica. La última aparece entre el 1\% y $2 \%$ de la población. Generalmente los portadores de una inversión no generan alteraciones fenotípicas; sin embargo, esta reorganización cromosómica puede llevar a gametos no balanceados, llevando a los portadores de la inversión a ser afectados por infertilidad por falla espermatogénica y abortos. (7). Fuera de esto, existe una variante que son las inversiones de novo que pueden generar severos efectos fenotípicos en el individuo, si el punto de inversión involucra regiones genéticas críticas, entre esas las más comunes son el retardo mental, anormalidades craneofaciales, atresia del meato auditivo y microtia según la región que se afecte (10).

Dentro de la secuencia de genes que se codifican en el cromosoma 5, se pueden observar diversas patologías asociadas a las inversiones, entre las que se encuentra el síndrome hipereosinofílico (inversión pericéntrica), que consiste en una entidad rara en la que se evidencia un conteo absoluto de eosinófilos de más de $15.000 \mathrm{uL}$ por mas de 6 meses asociado a daño de órgano, eosinofilia reactiva, cánceres hematológicos o fascitis eosinofílica. En esta entidad se ha observado una asociación con la inversión en el cromosoma 5 ya que este codifica para el factor de crecimiento derivado de plaquetas receptor beta, generando una mutación y por ende la enfermedad descrita (11-12).

El Síndrome Hermansky-Pudlak es un grupo de desórdenes genéticos heterogéneos que son causados por una mutación en los genes que afectan la síntesis y función de los organelos asociados a los lisosomas, los cuales son ampliamente distribuidos en tejidos hematopoyéticos. Una de esas altera- ciones genéticas se ve presente en el Síndrome de Hermansky-Pudlak tipo 2 donde se ha evidenciado que una inversión en el cromosoma 5 realizaría disfunción plaquetaria y neutropenia generando que el paciente tenga susceptibilidad a infecciones virales y raramente linfohisticocitosis hemofagocítica como respuesta a una infección viral (13).

Además de las enfermedades previamente descritas asociadas a inversión del cromosoma 5 se ha evidenciado en la literatura asociación con neoplasias como, por ejemplo, la leucemia linfoblástica aguda (LLA), la cual se presenta en un tercio de todos los pacientes pediátricos con cáncer y se caracteriza por la proliferación y acumulación de blastos malignos en el hueso y en la sangre periférica, con un pico aproximado de incidencia entre 2 a 5 ańos de edad. Aunque se han realizado reportes de caso en el que se evidencia asociación con síndromes genéticos, realmente la causa de LLA permanece desconocida, pero por la gravedad de su comportamiento es necesario tener en cuenta la inversión del cromosoma 5 como prioridad en el estudio de la misma (14-15). Algunas de las enfermedades benignas a asociadas son las convulsiones familiares benignas neonatales, las cuales están asociadas a un patrón de herencia autosómica dominante, generando que se presenten convulsiones en el primer mes de vida y usualmente desaparecen a la edad de 6 meses (16). Existe otra enfermedad específicamente asociada al brazo q y a la inversión de este, ya que se codifica el receptor acoplado a proteína $G$ que afecta el descenso testicular, por lo cual se ha evidenciado en la literatura que al haber una inversión del cromosoma existe la posibilidad de generar criptorquidia bilateral y anormalidades fenotípicas (17). Finalmente, una de las enfermedades mas comúnmente asociada al cromosoma 5 es el Síndrome Cri-du-chat el cual es causado por haploinsuficiencia de los genes en la parte distal del brazo corto del cromosoma 5, generando algunas características como microcefalia, retraso en el desarrollo, y un sollozo parecido al maullido de un gato. La mayoría de pacientes con este síndrome se debe a una delección esporádica de novo, que está asociado a bajo riesgo de recurren- 
cia. Sin embargo, en algunos casos esta asociado a una monosomía 5p acompańado de trisomía $5 \mathrm{q}$ y esta combinación es virtualmente asociada a inversiones pericéntricas largas parentales, por lo cual siempre se debe realizar un cribado en el paciente como en los familiares (18).

Igualmente, es importante mencionar algunas de las enfermedades que se pueden generar por otra alteración cromosómica diferente de la inversión. Existen pocos reportes de duplicaciones en el brazo largo del cromosoma 5 en el que rara vez se evidencia una misma duplicación en los mismos segmentos, dando pie a generar retardo en el crecimiento, comportamiento hiperkinético, retraso en el habla y psicomotor (19).

Luego de exponer las enfermedades más comunes $y$ algunas severas que se puede generar por inversión del cromosoma 5, este reporte de caso es una demostración de la normalidad cognitiva y de desarrollo en un paciente con inversión de novo en este cromosoma. Esto se puede explicar dado que el alelo normal presente en el cromosoma sin invertir es suficiente para compensar las alteraciones de cromosoma afectado. Es decir el cromosoma nuevo esta compensado (20-23).

Finalmente, es importante destacar que el permiso para el uso de la información utilizada en este reporte de caso, ha sido dado por la Unidad de Genética Médica donde el paciente ha sido evaluado. De la misma forma se contó con el respectivo consentimiento informado.

\section{Conflicto de intereses}

Los autores declaran que no tienen conflicto de interés.

\section{Referencias}

1. Chang HP, Chion JY, Chen JY, Su PH. Prenatal cytogenetic diagnosis in Taiwan: a nationwide population-based study. The Journal of Maternal-Fetal \& Neonatal Medicine [Internet]. 2016 [citado 01 Feb 2017];0(0):1-8. Disponible en: http://doi.org/10.1080/14767058.2016.1255191.

2. Petersson K, Lindkvist M, Persson M, Conner P, Åhman A, Mogren I. Prenatal diagnosis in Sweden 2011 to 2013-a register-based study. BMC Pregnancy and Childbirth [Internet]. 2016 [citado 01 Feb 2017];16(1):1-13. Disponible en: http:// doi.org/10.1186/s12884-016-1165-8.

3. Cheng JF. Chromosome 5. Encyclopedia of life sciences [Internet]. 2006 [citado $17 \mathrm{Feb}$ 2017];0(0):1-8. Disponible en: http://onlinelibrary.wiley.com/doi/10.1038/npg.els.0005814/ abstract.

4. Mandal A. ¿Cuál es el Cromosoma 5 ? NEWS Med [Internet]. 2014 [citado 10 Feb 2017];0(0):1-5. Disponible en: http:// www.news-medical.net/health/What-is-Chromosome-5-(Spanish).aspx.

5. Schmutz J, Martin J, Terry A, Couronne O, Grimwood J, Lowry $S$, et al. The DNA sequence and comparative analysis of human chromosome 5. Nature [Internet].2004 [citado 08 Feb 2017];431(1):268-274. Disponible en: http://www.ncbi. nlm.nih.gov/pubmed/15372022

6. Kaffenberger B. Inversion. Understanding cromosomas disorders [Internet]. 2014 [citado 08 Feb 2017];0(0):1-12. Disponible en: http://www.rarechromo.org/information/Other/ Inversions\%20FTNW.pdf

7. Bernicot I, Dechanet C, MacE A, Hedon B, Hamamah S, Pellestor F, et al. Predictive value of sperm-FISH analysis on the outcome of preimplantation genetic diagnosis (PGD) for a pericentric inversion inv5(p15.3q11.2) carrier. Hum Reprod [Internet]. 2010 [citado 10 Feb 2017];25(7):18181823. Disponible en: http://www.fertstert.org/article/S00150282(09)03005-2/abstract

8. Conditions G. Genetics home reference. Genet Home Ref [Internet]. 2017[citado 05 Feb 2017];0(0):1-7. Disponible en: https://ghr.nlm.nih.gov/chromosome/5.pdf.

9. Siddiqi R, Gilbert F. Chromosome 5. Genetic Test [Internet]. 2004 (citado 11 Feb 2017).7(2): 16987. Disponible en: http://online.liebertpub.com/doi/ abs/10.1089/109065703322146902

10. Evans J a. Diaphragmatic defects and limb deficiencies - taking sides. Am J Med Genet A [Internet]. 2007 (citado 09 Feb 2017);143(18):2106-2112. Disponible en: https://www.ncbi. nlm.nih.gov/pubmed/17701998

11. Saultz JN, Kaffenberger BH, Taylor M, Heerema NA, Klisovic R. Novel Chromosome 5 Inversion Associated With PDGFRB Rearrangement in Hypereosinophilic Syndrome. JAMA Dermatology [Internet]. 2016 (citado 12 Feb 2017);152(12):1391-1392. Disponible en: http://archderm. jamanetwork.com/article.aspx?doi $=10.1001 /$ jamadermatol.2016.3175 
12. Ferguson JS, Bosworth J, Min T, Mercieca J, Holden CA Eosinophilic fasciitis associated with hypereosinophilia, abnormal bone-marrow karyotype and inversion of chromosome 5. Clin Exp Dermatol [Internet]. 2014 (citado 11 Feb 2017);39(2):150-153. Disponible en: https://www.ncbi.nlm. nih.gov/pubmed/24237445

13. Jones ML, Murden SL, Brooks C, Maloney V, Manning RA, Gilmour KC, et al. Disruption of AP3B1 by a chromosome 5 inversion: 1 new disease mechanism in Hermansky-Pudlak syndrome type 2. BMC Med Genet [Internet]. 2013 (citado 11 Feb 2017);0(0):1-14. Disponible en: http://link.springer. com/article/10.1186/1471-2350-14-42

14. De Oliveira FM, Scrideli CA, Queiroz RG, Tone LG. Acute lymphoblastic leukemia with $\operatorname{inv}(5)(\mathrm{q} 13 \mathrm{q} 31)$ in a pediatric patient. Cancer Genet Cytogenet [Internet]. 2006 (citado 11 Feb 2017);165(1):81-82. Disponible en: http://www.ncbi. nlm.nih.gov/pubmed/16490602.

15. Butcher BW, Wilson KS, Kroft SH, Collins RH. Acute leukemia with B-lymphoid and myeloid differentiation associated with an inv $(5)(\mathrm{q} 13 \mathrm{q} 33)$ in an adult patient. Cancer Genet Cytogenet. 2005 (citado 11 Feb 2017);157(1):62-66. Disponible en: https:/www.ncbi.nlm.nih.gov/pubmed/15676149

16. Concolino D, Iembo MA, Rossi E, Giglio S, Coppola G, Miraglie $\mathrm{E}$, et al. Familial pericentric inversion of chromosome 5 in a family with benign neonatal convulsions. J Med Genet [Internet]. 2002 (citada 11 Feb 2017);39(3):214-216. Disponible en: http://www.ncbi.nlm.nih.gov/entrez/query. fcgi? cmd $=$ Retrieve $\& \mathrm{db}=$ PubMed $\&$ dopt=Citation \&list uids $=11897828$.

17. Dutta MK, Gundgurthi A, Garg MK, Pakhetra R. Cryptorchidism due to chromosome $5 \mathrm{q}$ inversion duplication. J Assoc Physicians India [Internet]. 2013 (citada 11 Feb 2017);61(1):925-927. Disponible en: https://www.ncbi.nlm. nih.gov/pubmed/24968555

18. Ohnuki Y, Torii C, Kosaki R, Yagihashi T, Sago H, Hayashi K, et al. Cri-du-chat syndrome cytogenetically cryptic recombination aneusomy of chromosome 5: Implications in recurrence risk estimation. Mol Syndromol [Internet]. 2010 (citada 12 Feb 2017);1(2):95-98. Disponible en: https://www.ncbi.nlm. nih.gov/pubmed/21045963

19. Schmidt T, Bartels I, Liehr T, Burfeind P, Zoll B, Shoukier M. A family with an inverted tandem duplication 5q22.1q23.2. Cytogenet Genome Res [Internet]. 2012 (citado 11 Feb 2017); 139(1):65-70. Disponible en: https://www.karger. com/Article/Abstract/342914

20. Pierce BA. Genética un enfoque conceptual [Internet]. 5 ed. Estados Unidos: Panamericana; 2014. [Citado 11 Feb 2017]. Disponible en: https://books.google.es/books?id=ALR9bgLtFhYC\&printsec=frontcover\&hl=es\&source=gbs_ge_sum mary_r\&cad $=0 \# v=$ onepage $\& q \& f=$ false

21. Bernal Luz Mery, López Greizy. Diagnóstico prénatal: retrospectiva. Nova. 2014; $12(21)$ : 23-36.

22. Almonacid Urrego Carmen Cecilia, Camarillo Romero María del Socorro, Gil Murcia Zulay, Medina Medina Claudia Yasmin, Rebellón Marulanda Jennifer Viviana, Mendieta Zerón
Hugo. Evaluación de factores de riesgo asociados a enfermedad cardiovascular en jóvenes universitarios de la Localidad Santafé en Bogotá, Colombia. Nova. 2016; 14(25): 9-17.

23. González Devia Johanna L., Monroy Romero Paola A., Almonacid Urrego Carmen C.. Homocisteína y otros factores de riesgo cardiovascular en niños de educación básica primaria del Colegio Distrital Manuel Elkin Patarroyo, Bogotá, D.C. Colombia. Estudio piloto. Nova. 2017 ; 15( 27 ): 103-117. 